\title{
EXISTENCE OF POSITIVE SOLUTIONS FOR SOME POLYHARMONIC NONLINEAR EQUATIONS IN $\mathbb{R}^{n}$
}

\author{
HABIB MÂAGLI AND MALEK ZRIBI \\ Received 25 December 2004; Accepted 1 March 2005
}

We will study the following polyharmonic nonlinear elliptic equation $(-\Delta)^{m} u+f(\cdot, u)=$ 0 in $\mathbb{R}^{n}, n>2 m$. Under appropriate conditions on the nonlinearity $f(x, t)$, related to a class of functions called $m$-Green-tight functions, we give some existence results for the above equation.

Copyright (c) 2006 H. Mâagli and M. Zribi. This is an open access article distributed under the Creative Commons Attribution License, which permits unrestricted use, distribution, and reproduction in any medium, provided the original work is properly cited.

\section{Introduction}

In this paper, we deal with the higher order elliptic equation

$$
(-\Delta)^{m} u=f(\cdot, u), \quad \text { in } \mathbb{R}^{n},
$$

where $m$ is a positive integer such that $n>2 m$.

In the case $m=1,(1.1)$ contains several well-known types which have been studied extensively by many authors (see for example $[1-3,8,9,11,12,14]$ and the references therein). Their basic tools are essentially some properties of functions belonging to the classical Kato class $K_{n}\left(\mathbb{R}^{n}\right)$ and the subclass of Green-tight functions $K_{n}^{\infty}\left(\mathbb{R}^{n}\right)$ (some properties pertaining to these classes can be found in $[1,4,14])$.

In this paper, we are concerned with the high order. Our purpose is two folded. One is to extend the Kato class $K_{n}\left(\mathbb{R}^{n}\right)$ and the subclass $K_{n}^{\infty}\left(\mathbb{R}^{n}\right)$ to the order $m \geq 2$. The second purpose is to investigate the existence of positive solutions for (1.1). The outline of the paper is as follows. The existence results are given in Sections 3, 4 and 5. In Section 2, we give the explicit formula of the Green function $G_{m, n}(x, y)$ of $(-\Delta)^{m}$ in $\mathbb{R}^{n}$. Namely, for each $x, y$ in $\mathbb{R}^{n}$

$$
G_{m, n}(x, y)=k_{m, n} \frac{1}{|x-y|^{n-2 m}}
$$

Hindawi Publishing Corporation

Abstract and Applied Analysis

Volume 2006, Article ID 76582, Pages 1-24

DOI 10.1155/AAA/2006/76582 


\section{Solutions of polyharmonic equations in $\mathbb{R}^{n}$}

where $k_{m, n}$ is a positive constant which will be precised later. The $3 G$-Theorem proved in [13] for the case $m=1$, is also valid for every $m$. Indeed, for each $x, y, z$ in $\mathbb{R}^{n}$, we have

$$
\frac{G_{m, n}(x, z) G_{m, n}(z, y)}{G_{m, n}(x, y)} \leq 2^{n-2 m-1}\left[G_{m, n}(x, z)+G_{m, n}(z, y)\right]
$$

This 3G-Theorem will be useful to state our existence results.

Next, we study the Kato class $K_{m, n}\left(\mathbb{R}^{n}\right)$ defined as follows.

Definition 1.1. A Borel measurable function $\varphi$ in $\mathbb{R}^{n}(n>2 m)$, belongs to the Kato class $K_{m, n}\left(\mathbb{R}^{n}\right)$ if

$$
\lim _{\alpha \rightarrow 0}\left(\sup _{x \in \mathbb{R}^{n}} \int_{|x-y| \leq \alpha} \frac{|\varphi(y)|}{|x-y|^{n-2 m}} d y\right)=0 .
$$

Indeed, first we prove some properties of functions belonging to this class similar to those established in $[1,4]$. In particular, we have the following characterization

$$
\varphi \in K_{m, n}\left(\mathbb{R}^{n}\right) \Longleftrightarrow \lim _{t \rightarrow 0}\left(\sup _{x \in \mathbb{R}^{n}} \int_{0}^{t} s^{m-1} \int_{\mathbb{R}^{n}} p(s, x, y)|\varphi(y)| d y d s\right)=0
$$

where $p(t, x, y)=\left(1 /(4 \pi t)^{n / 2}\right) \exp \left(-|x-y|^{2} / 4 t\right)$, for $t \in(0, \infty)$ and $x, y \in \mathbb{R}^{n}$, is the density of the Gauss semi-group on $\mathbb{R}^{n}$.

Secondly, we study a subclass of $K_{m, n}\left(\mathbb{R}^{n}\right)$ denoted by $K_{m, n}^{\infty}\left(\mathbb{R}^{n}\right)$ and defined by the following.

Definition 1.2. A Borel measurable function $\varphi$ belongs to the class $K_{m, n}^{\infty}\left(\mathbb{R}^{n}\right)$ and it is called $m$-Green-tight function if $\varphi \in K_{m, n}\left(\mathbb{R}^{n}\right)$ and satisfies

$$
\lim _{M \rightarrow \infty}\left(\sup _{x \in \mathbb{R}^{n}} \int_{|y| \geq M} \frac{|\varphi(y)|}{|x-y|^{n-2 m}} d y\right)=0 .
$$

In particular, we characterize the class $K_{m, n}^{\infty}\left(\mathbb{R}^{n}\right)$ as follows.

Theorem 1.3. Let $\varphi \in \mathscr{B}^{+}\left(\mathbb{R}^{n}\right),(n>2 m)$. Then the following assertions are equivalent

(1) $\varphi \in K_{m, n}^{\infty}\left(\mathbb{R}^{n}\right)$.

(2) The m-potential of $\varphi, V \varphi(x):=\int_{\mathbb{R}^{n}} G_{m, n}(x, y) \varphi(y) d y$ is in $C_{0}^{+}\left(\mathbb{R}^{n}\right)$.

This Theorem improves the result of Zhao in [14], for the case $m=1$. A more fine characterization will be given in the radial case.

One can easily check that $L^{1}\left(\mathbb{R}^{n}\right) \cap K_{m, n}\left(\mathbb{R}^{n}\right) \subset K_{m, n}^{\infty}\left(\mathbb{R}^{n}\right)$. Also we show that for $p>$ $n / 2 m$ and $\lambda<2 m-n / p<\mu$, we have

$$
\frac{L^{p}\left(\mathbb{R}^{n}\right)}{(1+|\cdot|)^{\mu-\lambda}|\cdot|^{\lambda}} \subset K_{m, n}^{\infty}\left(\mathbb{R}^{n}\right)
$$

and we precise the behaviour of the $m$-potential of functions in this class. 
In Section 3, we are interested in the following polyharmonic problem

$$
\begin{gathered}
(-\triangle)^{m} u+u \varphi(\cdot, u)=0, \quad \text { in } \mathbb{R}^{n} \text { (in the sense of distributions) } \\
\lim _{|x| \rightarrow \infty} u(x)=c>0 .
\end{gathered}
$$

The function $\varphi$ is required to verify the following assumptions.

$\left(\mathrm{H}_{1}\right) \varphi$ is a nonnegative measurable function on $\mathbb{R}^{n} \times(0, \infty)$.

$\left(\mathrm{H}_{2}\right)$ For each $\lambda>0$, there exists a nonnegative function $q_{\lambda} \in K_{m, n}^{\infty}\left(\mathbb{R}^{n}\right)$ with $\alpha_{q_{\lambda}} \leq 1 / 2$ (see (1.24)) and such that for each $x \in \mathbb{R}^{n}$, the mapping $t \rightarrow t\left(q_{\lambda}(x)-\varphi(x, t)\right)$ is continuous and nondecreasing on $[0, \lambda]$.

Under these hypotheses, we give an existence result for the problem (1.8). In fact, we will prove the following theorem.

Theorem 1.4. Assume $\left(H_{1}\right)$ and $\left(H_{2}\right)$. Then the problem (1.8) has a positive continuous solution $u$ in $\mathbb{R}^{n}$ satisfying for each $x \in \mathbb{R}^{n}, c / 2 \leq u(x) \leq c$.

To establish this result, we use a potential theory approach. In particular, we prove that if the function $q \in K_{m, n}^{\infty}\left(\mathbb{R}^{n}\right)$ is sufficiently small and $f$ is a nonnegative function on $\mathbb{R}^{n}$, then the equation

$$
(-\triangle)^{m} u+q u=f
$$

has a positive solution on $\mathbb{R}^{n}$. In [6], Grunau and Sweers gave a similar result in the unit ball of $\mathbb{R}^{n}$, with operators perturbed by small lower order terms:

$$
(-\triangle)^{m} u+\sum_{|k|<2 m} a_{k}(u) D^{k} u=f .
$$

In the case $m=1$, the problem (1.8) has been studied by Mâagli and Masmoudi in [7,8], where they gave an existence and an uniqueness result in both bounded and unbounded domain $\Omega$.

In Section 4, we are concerned with the following polyharmonic problem

$$
\begin{gathered}
(-\triangle)^{m} u=f(\cdot, u), \quad \text { in } \mathbb{R}^{n} \text { (in the sense of distributions) } \\
\lim _{|x| \rightarrow \infty} u(x)=0 .
\end{gathered}
$$

Here $f$ is required to satisfy the following assumptions.

$\left(\mathrm{H}_{3}\right) f$ is a nonnegative measurable function on $\mathbb{R}^{n} \times(0, \infty)$, continuous with respect to the second variable.

$\left(\mathrm{H}_{4}\right)$ There exist a nonnegative function $p$ in $\mathbb{R}^{n}$ such that

$$
0<\alpha_{0}:=\int_{\mathbb{R}^{n}} \frac{p(y)}{(|y|+1)^{2(n-2 m)}} d y<\infty
$$

and a nonnegative function $q \in K_{m, n}^{\infty}\left(\mathbb{R}^{n}\right)$ such that for $x \in \mathbb{R}^{n}$ and $t>0$

$$
p(x) h(t) \leq f(x, t) \leq q(x) g(t)
$$


4 Solutions of polyharmonic equations in $\mathbb{R}^{n}$

where $h$ is a nonnegative nondecreasing measurable function on $[0, \infty)$ satisfying

$$
m_{0}:=\frac{1}{k_{m, n} \alpha_{0}}<h_{0}:=\liminf _{t \rightarrow 0^{+}} \frac{h(t)}{t} \leq \infty
$$

and $g$ is a nonnegative measurable function locally bounded on $[0, \infty)$ satisfying

$$
0 \leq g^{\infty}:=\limsup _{t \rightarrow \infty} \frac{g(t)}{t}<M_{0}:=\frac{1}{\|V q\|_{\infty}} .
$$

By using a fixed point argument, we will state the following existence result.

Theorem 1.5. Assume $\left(H_{3}\right)$ and $\left(H_{4}\right)$. Then the problem (1.11) has a positive continuous solution $u$ in $\mathbb{R}^{n}$ satisfying for each $x \in \mathbb{R}^{n}$,

$$
\frac{a}{(|x|+1)^{n-2 m}} \leq u(x) \leq b V q(x),
$$

where $a, b$ are positive constants.

This result follows up the one of Dalmasso (see [5]), who studied the problem (1.11) in the unit ball $B$, with more restrictive conditions on the function $f$. Indeed, he assumed that $f$ is nondecreasing with respect to the second variable and satisfies

$$
\lim _{t \rightarrow 0^{+}} \min _{x \in \bar{B}} \frac{f(x, t)}{t}=+\infty, \quad \lim _{t \rightarrow+\infty} \max _{x \in \bar{B}} \frac{f(x, t)}{t}=0 .
$$

He proved the existence of a positive solution and he gave also an uniqueness result for positive radial solution when $f(x, t)=f(|x|, t)$.

When $m=1$, similar conditions, but more restrictive, on the nonlinearity $f$ have been adopted by Mâagli and Masmoudi in [8]. In fact in [8], the authors studied (1.11) in an unbounded domain $D$ of $\mathbb{R}^{n}, n \geq 3$, with compact nonempty boundary $\partial D$ and gave an existence result as Theorem 1.5.

On the other hand, Brezis and Kamin proved in [3], the existence and the uniqueness of a positive solution for the problem

$$
\begin{gathered}
-\Delta u=\rho(x) u^{\alpha} \quad \text { in } \mathbb{R}^{n}, \\
\liminf _{|x| \rightarrow \infty} u(x)=0,
\end{gathered}
$$

with $0<\alpha<1$ and $\rho$ is a nonnegative measurable function satisfying some appropriate conditions. We improve in this section the result of Brezis and Kamin in [3] and the one of Mâagli and Masmoudi in [8].

In Section 5, we will study the existence of solutions to the following polyharmonic problem

$$
\begin{gathered}
(-\triangle)^{m} u=f(\cdot, u), \quad \text { in } \mathbb{R}^{n} \text { (in the sense of distributions) } \\
u(x)>0, \quad \text { in } \mathbb{R}^{n},
\end{gathered}
$$

under the following assumptions on the nonlinearity $f$. 
$\left(\mathrm{H}_{5}\right) f$ is a nonnegative measurable function on $\mathbb{R}^{n} \times(0, \infty)$, continuous with respect to the second variable on $(0, \infty)$.

$\left(\mathrm{H}_{6}\right) f(x, t) \leq q(x, t)$, where $q$ is a nonnegative measurable function on $\mathbb{R}^{n} \times(0, \infty)$ such that the function $t \rightarrow q(x, t)$ is nondecreasing on $(0, \infty)$.

$\left(\mathrm{H}_{7}\right)$ There exists a constant $c>0$ such that $q(\cdot, c) \in K_{m, n}^{\infty}\left(\mathbb{R}^{n}\right)$ and

$$
\|V(q(\cdot, c))\|_{\infty}<c
$$

Put $c^{*}=c-\|V(q(\cdot, c))\|_{\infty}$. We give in this section the following existence result.

Theorem 1.6. Assume $\left(H_{5}\right),\left(H_{6}\right)$, and $\left(H_{7}\right)$. Then for each $\delta \in\left(0, c^{*}\right]$, the problem (1.19) has a positive continuous solution $u$ in $\mathbb{R}^{n}$ satisfying for each $x \in \mathbb{R}^{n}$

$$
\begin{aligned}
& \delta \leq u(x) \leq c \\
& \lim _{|x| \rightarrow \infty} u(x)=\delta .
\end{aligned}
$$

If $m=1$, Yin gave in [11] an existence result of the following problem

$$
\begin{aligned}
\triangle u+f(x, u) & =0, \quad \text { in } G_{B}, \\
u(x) & >0,
\end{aligned}
$$

where $G_{B}=\left\{x \in \mathbb{R}^{n},|x|>B\right\}$, for some $B \geq 0$. His method relies on the technique of radial super/subsolutions. Our approach is different, in fact we will use a fixed point argument. We improve the result of Yin under more general assumptions (see Remark 5.3).

In order to simplify our statements, we define some convenient notations.

\section{Notations.}

(i) $\mathscr{B}\left(\mathbb{R}^{n}\right)$ denotes the set of Borel measurable functions in $\mathbb{R}^{n}$ and $\mathscr{B}^{+}\left(\mathbb{R}^{n}\right)$ the set of nonnegative ones.

(ii) $C_{0}\left(\mathbb{R}^{n}\right):=\left\{w\right.$ continuous on $\mathbb{R}^{n}$ and $\left.\lim _{|x| \rightarrow \infty} w(x)=0\right\}$ and $C_{0}^{+}\left(\mathbb{R}^{n}\right)$ the set of nonnegative ones.

(iii) For $\varphi \in \mathscr{B}^{+}\left(\mathbb{R}^{n}\right)$, we put the $m$-potential of $\varphi$ on $\mathbb{R}^{n}$ by

$$
V \varphi(x):=V_{m, n} \varphi(x)=\int_{\mathbb{R}^{n}} G_{m, n}(x, y) \varphi(y) d y=k_{m, n} \int_{\mathbb{R}^{n}} \frac{\varphi(y)}{|x-y|^{n-2 m}} d y .
$$

(iv) For $\varphi \in \mathscr{B}^{+}\left(\mathbb{R}^{n}\right)$, we put

$$
\alpha_{\varphi}=\sup _{x, y \in \mathbb{R}^{n}} \int_{\mathbb{R}^{n}} \frac{G_{m, n}(x, z) G_{m, n}(z, y)}{G_{m, n}(x, y)}|\varphi(z)| d z .
$$

(v) Let $\lambda \in \mathbb{R}$, we denote by $\lambda^{+}=\max (\lambda, 0)$.

(vi) Let $f$ and $g$ be two positive functions on a set $S$.

We call $f \sim g$, if there is $c>0$ such that

$$
\frac{1}{c} g(x) \leq f(x) \leq c g(x) \quad \forall x \in S .
$$


We call $f \preceq g$, if there is $c>0$ such that

$$
f(x) \leq \operatorname{cg}(x) \quad \forall x \in S
$$

The following properties will be used several times: for $s, t \geq 0$, we have

$$
\begin{gathered}
\min (s, t)=s \wedge t \sim \frac{s t}{s+t}, \\
(s+t)^{p} \sim s^{p}+t^{p}, \quad p \in \mathbb{R}^{+} .
\end{gathered}
$$

\section{Properties of the Kato class}

In this section, we characterize functions belonging to the Kato class $K_{m, n}\left(\mathbb{R}^{n}\right)$ and the subclass $K_{m, n}^{\infty}\left(\mathbb{R}^{n}\right)$ of $m$-Green-tight functions and we prove Theorem 1.3. We recall that throughout this paper, we are concerned with $n>2 m$.

We set $p(t, x, y)=\left(1 /(4 \pi t)^{n / 2}\right) \exp \left(-|x-y|^{2} / 4 t\right)$, for $t \in(0, \infty)$ and $x, y \in \mathbb{R}^{n}$, the density of the Gauss semi-group on $\mathbb{R}^{n}$. By a simple computation, we obtain that the Green function of $(-\Delta)^{m}$ in $\mathbb{R}^{n}$, for each $m \geq 1$, is given by

$$
G_{m, n}(x, y)=\frac{1}{(m-1) !} \int_{0}^{\infty} s^{m-1} p(s, x, y) d s, \quad \text { for } x, y \text { in } \mathbb{R}^{n}
$$

Then we have the following explicit expression

$$
G_{m, n}(x, y)=k_{m, n} \frac{1}{|x-y|^{n-2 m}}, \quad \text { for } x, y \text { in } \mathbb{R}^{n}
$$

where $k_{m, n}=\Gamma(n / 2-m) / 4^{m} \pi^{n / 2}(m-1) !$.

2.1. The class $K_{m, n}\left(\mathbb{R}^{n}\right)$. We will study properties of functions belonging to $K_{m, n}\left(\mathbb{R}^{n}\right)$. First we remark the following comparison on the classes $K_{j, n}\left(\mathbb{R}^{n}\right)$, for $j \geq 1$.

Remark 2.1. Let $j, m \in \mathbb{N}$ such that $1 \leq j \leq m$, then we have for each $n>2 m$

$$
K_{n}\left(\mathbb{R}^{n}\right):=K_{1, n}\left(\mathbb{R}^{n}\right) \subseteq K_{j, n}\left(\mathbb{R}^{n}\right) \subseteq K_{m, n}\left(\mathbb{R}^{n}\right),
$$

where $K_{n}\left(\mathbb{R}^{n}\right)$ is the classical Kato class introduced in [1].

Example 2.2. Let $\varphi \in \mathscr{B}\left(\mathbb{R}^{n}\right)$. Suppose that for $p>n / 2 m$, we have

$$
\sup _{x \in \mathbb{R}^{n}} \int_{|x-y| \leq 1}|\varphi(y)|^{p} d y<\infty \text {. }
$$

Then by the Hölder inequality, we conclude that $\varphi \in K_{m, n}\left(\mathbb{R}^{n}\right)$.

In particular, we have that for $p>n / 2 m, L^{p}\left(\mathbb{R}^{n}\right) \subset K_{m, n}\left(\mathbb{R}^{n}\right)$.

To establish the characterization (1.5) of the Kato class $K_{m, n}\left(\mathbb{R}^{n}\right)$, we need the following lemmas. 
Lemma 2.3. For each $t>0$ and $x, y \in \mathbb{R}^{n}$, we have

$$
\int_{0}^{t} s^{m-1} p(s, x, y) d s \preceq G_{m, n}(x, y) .
$$

Moreover, for $|x-y| \leq 2 \sqrt{t}$, we have that

$$
G_{m, n}(x, y) \preceq \int_{0}^{t} s^{m-1} p(s, x, y) d s .
$$

Proof. Let $t>0$ and $x, y \in \mathbb{R}^{n}$. Then (2.5) follows immediately from (2.1).

If we suppose further that $|x-y| \leq 2 \sqrt{t}$, then we have

$$
\begin{aligned}
\int_{0}^{t} s^{m-1} p(s, x, y) d s & =c \int_{0}^{t} s^{m-n / 2-1} \exp \left(-\frac{|x-y|^{2}}{4 s}\right) d s \\
& =\frac{c}{|x-y|^{n-2 m}} \int_{|x-y|^{2} / 4 t}^{\infty} r^{n / 2-m-1} e^{-r} d r \\
& \geq \frac{c}{|x-y|^{n-2 m}} \int_{1}^{\infty} r^{n / 2-m-1} e^{-r} d r \\
& =c G_{m, n}(x, y),
\end{aligned}
$$

where the letter $c$ is a positive constant which may vary from line to line.

Lemma 2.4. Let $\varphi \in K_{m, n}\left(\mathbb{R}^{n}\right)$. Then for each compact $L \subset \mathbb{R}^{n}$, we have

$$
\sup _{x \in \mathbb{R}^{n}} \int_{x+L}|\varphi(y)| d y<\infty .
$$

In particular, we have $K_{m, n}\left(\mathbb{R}^{n}\right) \subset L_{\mathrm{loc}}^{1}\left(\mathbb{R}^{n}\right)$.

Proof. Let $\varphi \in K_{m, n}\left(\mathbb{R}^{n}\right)$, then by (1.4) there exists $\alpha>0$ such that

$$
\sup _{x \in \mathbb{R}^{n}} \int_{|x-y| \leq \alpha} \frac{|\varphi(y)|}{|x-y|^{n-2 m}} d y \leq 1 .
$$

Let $a_{1}, \ldots, a_{p} \in L$ such that $L \subseteq \bigcup_{1 \leq i \leq p} B\left(a_{i}, \alpha\right)$. Hence for each $x \in \mathbb{R}^{n}$, we have

$$
\begin{aligned}
\int_{x+L}|\varphi(y)| d y & \leq \sum_{i=1}^{p} \int_{B\left(x+a_{i}, \alpha\right)}|\varphi(y)| d y \\
& \leq \sum_{i=1}^{p} \alpha^{n-2 m} \int_{B\left(x+a_{i}, \alpha\right)} \frac{|\varphi(y)|}{\left|x+a_{i}-y\right|^{n-2 m}} d y \\
& \leq p \alpha^{n-2 m} .
\end{aligned}
$$

So, $\sup _{x \in \mathbb{R}^{n}} \int_{x+L}|\varphi(y)| d y<\infty$. 
8 Solutions of polyharmonic equations in $\mathbb{R}^{n}$

Proposition 2.5. Let $\varphi \in K_{m, n}\left(\mathbb{R}^{n}\right)$. Then for each fixed $\alpha>0$, we have

$$
\sup _{0 \leq t \leq 1}\left(\sup _{x \in \mathbb{R}^{n}} \int_{|x-y| \geq \alpha} t^{m-1} p(t, x, y)|\varphi(y)| d y\right):=M(\alpha)<\infty .
$$

Proof. Let $\varphi \in K_{m, n}\left(\mathbb{R}^{n}\right), 0<t \leq 1$. Let $\alpha>0$, then we have that

$$
\begin{aligned}
& \sup _{x \in \mathbb{R}^{n}} \int_{|x-y| \geq \alpha} t^{m-1} p(t, x, y)|\varphi(y)| d y \\
& \quad \preceq \frac{\exp \left(-\alpha^{2} / 8 t\right)}{t^{n / 2-m+1}} \sup _{x \in \mathbb{R}^{n}} \int_{\mathbb{R}^{n}} \exp \left(-\frac{|x-y|^{2}}{8}\right)|\varphi(y)| d y .
\end{aligned}
$$

So to prove (2.11), we need to show that

$$
\sup _{x \in \mathbb{R}^{n}} \int_{\mathbb{R}^{n}} \exp \left(-\frac{|x-y|^{2}}{8}\right)|\varphi(y)| d y<\infty .
$$

Indeed, using Lemma 2.4, we denote by

$$
c:=\sup _{x \in \mathbb{R}^{n}} \int_{x+B(0,1)}|\varphi(y)| d y<\infty .
$$

On the other hand, since any ball $B(0, k)$ of radius $k \geq 1$ in $\mathbb{R}^{n}$ can be covered by $\alpha(n):=$ $A_{n} k^{n}$ balls of radius 1 , where $A_{n}$ is a constant depending only on $n$ (see [4, page 67]), then there exist $a_{1}, a_{2}, \ldots, a_{\alpha(n)} \in B(0, k)$ such that

$$
B(0, k) \subset \bigcup_{1 \leq i \leq \alpha(n)} B\left(a_{i}, 1\right) .
$$

Hence for each $x \in \mathbb{R}^{n}$, we have

$$
\int_{x+B(0, k)}|\varphi(y)| d y \leq \sum_{i=1}^{\alpha(n)} \int_{B\left(x+a_{i}, 1\right)}|\varphi(y)| d y \leq c A_{n} k^{n},
$$

which implies that for each $x \in \mathbb{R}^{n}$,

$$
\begin{aligned}
\int_{\mathbb{R}^{n}} \exp \left(-\frac{|x-y|^{2}}{8}\right)|\varphi(y)| d y & \leq \sum_{k=0}^{\infty} \exp \left(-\frac{k^{2}}{8}\right) \int_{k \leq|x-y| \leq k+1}|\varphi(y)| d y \\
& \leq c A_{n} \sum_{k=0}^{\infty} \exp \left(-\frac{k^{2}}{8}\right)(k+1)^{n} \\
& <\infty
\end{aligned}
$$

Thus (2.13) holds. This ends the proof. 
Proposition 2.6. Let $\varphi \in B\left(\mathbb{R}^{n}\right)$. Then $\varphi \in K_{m, n}\left(\mathbb{R}^{n}\right)$ if and only if

$$
\lim _{t \rightarrow 0}\left(\sup _{x \in \mathbb{R}^{n}} \int_{0}^{t} s^{m-1} \int_{\mathbb{R}^{n}} p(s, x, y)|\varphi(y)| d y d s\right)=0 .
$$

Proof. Suppose $\varphi$ verifies (2.18), then from (2.6) we have that

$$
\int_{|x-y| \leq \alpha} \frac{|\varphi(y)|}{|x-y|^{n-2 m}} d y \preceq \int_{\mathbb{R}^{n}} \int_{0}^{\alpha^{2} / 4} s^{m-1} p(s, x, y)|\varphi(y)| d s d y,
$$

which implies that the function $\varphi$ satisfies (1.4).

Conversely, suppose that $\varphi \in K_{m, n}\left(\mathbb{R}^{n}\right)$. Let $\varepsilon>0$, then by (1.4), there exists $\alpha>0$ such that

$$
\sup _{x \in \mathbb{R}^{n}} \int_{|x-y| \leq \alpha} \frac{|\varphi(y)|}{|x-y|^{n-2 m}} d y \leq \varepsilon .
$$

Thus from (2.5) and (2.11), we deduce that for each $x \in \mathbb{R}^{n}$ and $t \leq 1$, we have

$$
\begin{aligned}
\int_{0}^{t} s^{m-1} & \int_{\mathbb{R}^{n}} p(s, x, y)|\varphi(y)| d y d s \\
\leq & \int_{|x-y| \leq \alpha} \int_{0}^{t} s^{m-1} p(s, x, y)|\varphi(y)| d y d s \\
& \quad+\int_{0}^{t} \int_{|x-y| \geq \alpha} s^{m-1} p(s, x, y)|\varphi(y)| d y d s \\
& \leq \int_{|x-y| \leq \alpha} \frac{|\varphi(y)|}{|x-y|^{n-2 m}} d y+t M(\alpha) \\
\preceq & \varepsilon+t M(\alpha) .
\end{aligned}
$$

This implies (2.18) and completes the proof.

2.2. The class $K_{m, n}^{\infty}\left(\mathbb{R}^{n}\right)$. We will characterize the subclass of $m$-Green-tight functions $K_{m, n}^{\infty}\left(\mathbb{R}^{n}\right)$. In fact, we will prove Theorem 1.3 and we give in particular a more precise characterization in the radial case.

Example 2.7. Let $p>n / 2 m$. Then $L^{p}\left(\mathbb{R}^{n}\right) \cap L^{1}\left(\mathbb{R}^{n}\right) \subset K_{m, n}^{\infty}\left(\mathbb{R}^{n}\right)$.

Proof of Theorem 1.3. Let $\varphi \in \mathscr{B}^{+}\left(\mathbb{R}^{n}\right)$. First we suppose that $\varphi \in K_{m, n}^{\infty}\left(\mathbb{R}^{n}\right)$, then using similar arguments as in the proof [9, Proposition 6], we obtain easily that $V \varphi \in C_{0}^{+}\left(\mathbb{R}^{n}\right)$.

Conversely we suppose that $V \varphi \in C_{0}^{+}\left(\mathbb{R}^{n}\right)$. Then, we aim at proving that $\varphi \in K_{m, n}^{\infty}\left(\mathbb{R}^{n}\right)$. So we divide the proof into two steps. 
Step 1. We will prove that $\varphi$ satisfies (2.18). Indeed it is clear from (2.1), that for each $x \in \mathbb{R}^{n}$, we have that

$$
\begin{aligned}
V \varphi(x)= & \frac{1}{(m-1) !} \int_{0}^{t} s^{m-1} \int_{\mathbb{R}^{n}} p(s, x, y) \varphi(y) d y d s \\
& +\frac{1}{(m-1) !} \int_{t}^{\infty} s^{m-1} \int_{\mathbb{R}^{n}} p(s, x, y) \varphi(y) d y d s \\
= & I_{1}(x)+I_{2}(x) .
\end{aligned}
$$

From the properties of the density $p(s, x, y)$, we deduce that $x \rightarrow I_{1}(x)$ and $x \rightarrow I_{2}(x)$ are nonnegative lower semi-continuous functions in $\mathbb{R}^{n}$. Then using the fact that $V \varphi \in$ $C_{0}^{+}\left(\mathbb{R}^{n}\right)$, we get that the function $x \rightarrow I_{1}(x)$ is also in $C_{0}^{+}\left(\mathbb{R}^{n}\right)$. So, for each $x \in \mathbb{R}^{n}$, the family $\left\{\int_{0}^{t} s^{m-1} \int_{\mathbb{R}^{n}} p(s, x, y) \varphi(y) d y d s, t>0\right\}$ is decreasing in $C_{0}^{+}\left(\mathbb{R}^{n}\right)$, which together with the fact that for each $x \in \mathbb{R}^{n}$,

$$
\lim _{t \rightarrow 0} \int_{0}^{t} s^{m-1} \int_{\mathbb{R}^{n}} p(s, x, y) \varphi(y) d y d s=0
$$

imply by Dini Lemma, that (2.18) is satisfied.

Step 2. We will prove that $\varphi$ satisfies (1.6). Let $\varepsilon>0$, then since $V \varphi \in C_{0}^{+}\left(\mathbb{R}^{n}\right)$, there exists $a>0$ such that for $|x| \geq a$, we have that $V \varphi(x) \leq \varepsilon$.

Let $M \geq 2 a$, then

$$
\begin{aligned}
\sup _{x \in \mathbb{R}^{n}} \int_{|y| \geq M} \frac{\varphi(y)}{|x-y|^{n-2 m}} d y & \leq \sup _{|x| \geq a} \int_{\mathbb{R}^{n}} \frac{\varphi(y)}{|x-y|^{n-2 m}} d y+\sup _{|x| \leq a} \int_{|y| \geq M} \frac{\varphi(y)}{|x-y|^{n-2 m}} d y \\
& \preceq \varepsilon+\int_{|y| \geq M} \frac{\varphi(y)}{|y|^{n-2 m}} d y .
\end{aligned}
$$

Now, since $V \varphi(0)<\infty$, we deduce that

$$
\lim _{M \rightarrow \infty} \int_{|y| \geq M} \frac{\varphi(y)}{|y|^{n-2 m}} d y=0
$$

Then (1.6) holds and this ends the proof.

For a nonnegative function $\rho$ in $K_{m, n}^{\infty}\left(\mathbb{R}^{n}\right)$, we denote by

$$
M_{\rho}:=\left\{\varphi \in \mathscr{B}\left(\mathbb{R}^{n}\right),|\varphi| \preceq \rho\right\} .
$$

Proposition 2.8. For a nonnegative function $\rho$ in $K_{m, n}^{\infty}\left(\mathbb{R}^{n}\right)$, the family of functions

$$
V\left(M_{\rho}\right):=\left\{V \varphi, \varphi \in M_{\rho}\right\}
$$

is uniformly bounded and equicontinuous in $C_{0}\left(\mathbb{R}^{n}\right)$ and consequently it is relatively compact in $C_{0}\left(\mathbb{R}^{n}\right)$. 
Proof. Let $\rho \in K_{m, n}^{\infty}\left(\mathbb{R}^{n}\right)$. Obviously, since each function $\varphi$ in $M_{\rho}$ is in $K_{m, n}^{\infty}\left(\mathbb{R}^{n}\right)$, we obtain by Theorem 1.3 that the family $V\left(M_{\rho}\right) \subset C_{0}\left(\mathbb{R}^{n}\right)$ and is uniformly bounded. Next, we prove the equicontinuity of functions in $V\left(M_{\rho}\right)$ on $\mathbb{R}^{n} \cup\{\infty\}$ by same arguments as in the proof of [9, Proposition 6]. Thus by Ascoli's Theorem the family $V\left(M_{\rho}\right)$ is relatively compact in $C_{0}\left(\mathbb{R}^{n}\right)$. This ends the proof.

Remark 2.9. We recall (see $[12,14]$ ) that for $m=1$ and $n \geq 3$, a radial function is in $K_{n}^{\infty}\left(\mathbb{R}^{n}\right)$ if and only if $\int_{0}^{\infty} r|\varphi(r)| d r<\infty$.

Similarly, we will give in the sequel a characterization of radial functions belonging to $K_{m, n}^{\infty}\left(\mathbb{R}^{n}\right)$.

Proposition 2.10. Let $\varphi$ be a radial function in $\mathbb{R}^{n}$, then $\varphi \in K_{m, n}^{\infty}\left(\mathbb{R}^{n}\right)$ if and only if

$$
\int_{0}^{\infty} r^{2 m-1}|\varphi(r)| d r<\infty
$$

In order to prove Proposition 2.10, we will use the following behaviour of the $m$ potential of radial functions on $\mathbb{R}^{n}$.

Proposition 2.11. Let $\varphi \in \mathscr{B}^{+}\left(\mathbb{R}^{n}\right)$ be a radial function on $\mathbb{R}^{n}$, then for $x \in \mathbb{R}^{n}$, we have

$$
V \varphi(x) \sim \int_{0}^{\infty} \frac{r^{n-1}}{(|x| \vee r)^{n-2 m}} \varphi(r) d r
$$

Proof. Let $\varphi \in \mathscr{B}^{+}\left(\mathbb{R}^{n}\right)$. First, we recall the well known results for $x, y \in \mathbb{R}^{n}$,

$$
\begin{gathered}
(n-2) k_{1, n} \int_{\mathbb{R}^{n}} \frac{\varphi(y)}{|x-y|^{n-2}} d y=\int_{0}^{\infty} \frac{r^{n-1}}{(|x| \vee r)^{n-2}} \varphi(r) d r \\
\int_{\mathbb{R}^{n}} \frac{d z}{|x-z|^{n-2}|y-z|^{n-2}}=\frac{c_{n}}{|x-y|^{n-4}} .
\end{gathered}
$$

This implies that there exists a constant $c>0$ such that

$$
\begin{aligned}
\int_{\mathbb{R}^{n}} \frac{\varphi(y)}{|x-y|^{n-4}} d y & =c \int_{0}^{\infty} r^{n-1} \varphi(r) \int_{0}^{\infty} \frac{t^{n-1}}{(|x| \vee t)^{n-2}(t \vee r)^{n-2}} d t d r \\
& \geq c \int_{0}^{\infty} r^{n-1} \varphi(r) \int_{|x| \vee r}^{\infty} \frac{1}{t^{n-3}} d t d r \\
& \geq \frac{c}{n-4} \int_{0}^{\infty} \frac{r^{n-1} \varphi(r)}{(|x| \vee r)^{n-4}} d r
\end{aligned}
$$

Hence, we obtain by recurrence that

$$
\int_{0}^{\infty} \frac{r^{n-1}}{(|x| \vee r)^{n-2 m}} \varphi(r) d r \preceq \int_{\mathbb{R}^{n}} \frac{\varphi(y)}{|x-y|^{n-2 m}} d y .
$$




\section{Solutions of polyharmonic equations in $\mathbb{R}^{n}$}

On the other hand, there exists a constant $\tilde{c}>0$ such that for each $x \in \mathbb{R}^{n}$,

$$
\begin{aligned}
\int_{\mathbb{R}^{n}} \frac{\varphi(y)}{|x-y|^{n-2 m}} d y & =\tilde{c} \int_{0}^{\infty} \int_{0}^{\pi} \frac{r^{n-1} \varphi(r)(\sin \theta)^{n-2}}{\left(|x|^{2}+r^{2}-2 r|x| \cos \theta\right)^{(n-2 m) / 2}} d \theta d r \\
& \leq \tilde{c} \int_{0}^{\infty} \int_{0}^{\pi} \frac{r^{n-1} \varphi(r)(\sin \theta)^{n-2}}{(|x| \vee r)^{n-2 m}(\sin \theta)^{n-2 m}} d \theta d r \\
& =\tilde{c}\left(\int_{0}^{\pi}(\sin \theta)^{2 m-2} d \theta\right)\left(\int_{0}^{\infty} \frac{r^{n-1} \varphi(r)}{(|x| \vee r)^{n-2 m}} d r\right) .
\end{aligned}
$$

Thus (2.29) holds.

Proof of Proposition 2.10. Suppose that $\varphi$ is a radial function in $K_{m, n}^{\infty}\left(\mathbb{R}^{n}\right)$, then by Theorem 1.3, $V \varphi(0)<\infty$ and so we deduce (2.28) from (2.29). have

Conversely, suppose that $\varphi$ satisfies (2.28). Let $\alpha>0$ and $t=|x|$, then by (2.29), we

$$
\begin{aligned}
\int_{|x-y| \leq \alpha} \frac{|\varphi(y)|}{|x-y|^{n-2 m}} d y & \preceq \int_{(t-\alpha)^{+}}^{t+\alpha} \frac{r^{n-1}}{(t \vee r)^{n-2 m}}|\varphi(r)| d r \\
& \leq \int_{(t-\alpha)^{+}}^{t+\alpha} r^{2 m-1}|\varphi(r)| d r .
\end{aligned}
$$

Let $\phi(s)=\int_{0}^{s} r^{2 m-1}|\varphi(r)| d r$, for $s \in[0, \infty]$. Using (2.28), we deduce that $\phi$ is a continuous function on $[0, \infty]$. This implies that

$$
\int_{(t-\alpha)^{+}}^{t+\alpha} r^{2 m-1}|\varphi(r)| d r=\phi(t+\alpha)-\phi\left((t-\alpha)^{+}\right)
$$

converges to zero as $\alpha \rightarrow 0$ uniformly for $t \in[0, \infty]$. So $\varphi$ verifies (1.4).

Next, we have by (2.29)

$$
\int_{|y| \geq M} \frac{|\varphi(y)|}{|x-y|^{n-2 m}} d y \preceq \int_{M}^{\infty} \frac{r^{n-1}}{(t \vee r)^{n-2 m}}|\varphi(r)| d r \leq \int_{M}^{\infty} r^{2 m-1}|\varphi(r)| d r
$$

which, using (2.28), tends to zero as $M \rightarrow \infty$ and so $\varphi$ verifies (1.6). This completes the proof.

We close this section by giving a class of functions included in $K_{m, n}^{\infty}\left(\mathbb{R}^{n}\right)$ and we precise the behaviour of the $m$-potential of functions in this class. We need the following lemma. 
Lemma 2.12. Let $\alpha>0$ and $a, b>0$ such that $a+b<n$. Then

$$
\int_{|x-y| \leq \alpha} \frac{d y}{|y|^{a}|x-y|^{b}} \preceq \alpha^{n-(a+b)}
$$

Proof. Let $\alpha>0$ and $a, b$ be nonnegative real numbers such that $a+b<n$. Then

$$
\begin{aligned}
\int_{|x-y| \leq \alpha} \frac{d y}{|y|^{a}|x-y|^{b}} & \leq \int_{(|x-y| \leq \alpha) \cap(|x-y| \leq|y|)} \frac{d y}{|x-y|^{a+b}}+\int_{(|y| \leq|x-y| \leq \alpha)} \frac{d y}{|y|^{a+b}} \\
& \preceq \int_{0}^{\alpha} r^{n-1-(a+b)} d r \\
& \preceq \alpha^{n-(a+b)} .
\end{aligned}
$$

Proposition 2.13. Let $p>n / 2 m$. Then for $\lambda<2 m-n / p<\mu$, we have

$$
\frac{L^{p}\left(\mathbb{R}^{n}\right)}{(1+|\cdot|)^{\mu-\lambda}|\cdot|^{\lambda}} \subset K_{m, n}^{\infty}\left(\mathbb{R}^{n}\right) .
$$

Proof. Let $p>n / 2 m$ and $q \geq 1$ such that $1 / p+1 / q=1$. Let $a$ be a function in $L^{p}\left(\mathbb{R}^{n}\right)$ and $\lambda<2 m-n / p<\mu$. First, we will prove that the function $\varphi(x):=a(x) /(1+|x|)^{\mu-\lambda}|x|^{\lambda}$ satisfies (1.4). Let $\alpha>0$, then by the Hölder inequality and Lemma 2.12, we have for $x \in \mathbb{R}^{n}$

$$
\begin{aligned}
\int_{|x-y| \leq \alpha} \frac{|\varphi(y)|}{|x-y|^{n-2 m}} d y & \leq\|a\|_{p}\left(\int_{|x-y| \leq \alpha} \frac{d y}{(1+|y|)^{(\mu-\lambda) q}|y|^{\lambda q|x-y|^{(n-2 m) q}}}\right)^{1 / q} \\
& \leq\|a\|_{p}\left(\int_{|x-y| \leq \alpha} \frac{d y}{|y|^{q \lambda^{+}}|x-y|^{(n-2 m) q}}\right)^{1 / q} \\
& \preceq\|a\|_{p} \alpha^{2 m-n / p-\lambda^{+}},
\end{aligned}
$$

which converges to zero as $\alpha \rightarrow 0$.

Secondly, we claim that $\varphi$ satisfies (1.6). To show the claim we use the Hölder inequality. Let $M>1$, then we have

$$
\begin{aligned}
\int_{|y| \geq M} \frac{|\varphi(y)|}{|x-y|^{n-2 m}} d y & \leq\|a\|_{p}\left(\int_{|y| \geq M} \frac{d y}{(1+|y|)^{(\mu-\lambda) q}|y|^{\lambda q}|x-y|^{(n-2 m) q}}\right)^{1 / q} \\
& \sim\|a\|_{p}\left(\int_{|y| \geq M} \frac{d y}{|y|^{\mu q|x-y|^{(n-2 m) q}}}\right)^{1 / q} \\
& =\|a\|_{p}(A(x))^{1 / q}
\end{aligned}
$$


14 Solutions of polyharmonic equations in $\mathbb{R}^{n}$

Furthermore

$$
\begin{aligned}
A(x) \preceq & \sup _{|x| \leq M / 2} \int_{|y| \geq M} \frac{d y}{|y|^{(n-2 m+\mu) q}} \\
& +\sup _{|x| \geq M / 2} \frac{1}{|x|^{\mu q}} \int_{(|y| \geq M) \cap(|x-y| \leq|x| / 2)} \frac{d y}{|x-y|^{(n-2 m) q}} \\
& +\sup _{|x| \geq M / 2} \frac{1}{|x|^{(n-2 m) q}} \int_{(|y| \geq M) \cap(|x| / 2 \leq|x-y| \leq 2|x|)} \frac{d y}{|y|^{\mu q}} \\
& +\sup _{|x| \geq M / 2} \int_{(|y| \geq M) \cap(|x-y| \geq 2|x|)} \frac{d y}{|x-y|^{(n-2 m+\mu) q}} \\
& \frac{1}{M^{(n-2 m+\mu) q-n}}+\sup _{|z| \geq M / 2} \frac{\log (3|z| / M)}{|z|^{(n-2 m) q}},
\end{aligned}
$$

which converges to zero as $M \rightarrow \infty$. This ends the proof.

Remark 2.14. It is obvious to see that for each $\varphi \in \mathscr{B}^{+}\left(\mathbb{R}^{n}\right)$, we have

$$
\frac{k_{m, n}}{(|x|+1)^{n-2 m}} \int_{\mathbb{R}^{n}} \frac{\varphi(y)}{(|y|+1)^{n-2 m}} d y \leq V \varphi(x) .
$$

We precise in the following, some upper estimates on the $m$-potential of functions in the class $L^{p}\left(\mathbb{R}^{n}\right) /(1+|\cdot|)^{\mu-\lambda}|\cdot|^{\lambda}$. Indeed, put for a nonnegative function $a \in L^{p}\left(\mathbb{R}^{n}\right)$ and $x \in \mathbb{R}^{n}$

$$
W a(x):=V\left(\frac{a}{(1+|\cdot|)^{\mu-\lambda}|\cdot| \lambda}\right)(x)=\int_{\mathbb{R}^{n}} G_{m, n}(x, y) \frac{a(y)}{(1+|y|)^{\mu-\lambda}|y|^{\lambda}} d y .
$$

Then we have the following.

Proposition 2.15. Let $p>n / 2 m$ and $\lambda<2 m-n / p<\mu$. Then there exists $c>0$ such that for each nonnegative function $a \in L^{p}\left(\mathbb{R}^{n}\right)$ and $x \in \mathbb{R}^{n}$, we have the following estimates

$$
W a(x) \leq c\|a\|_{p} \begin{cases}\frac{1}{(1+|x|)^{n-2 m} \log (|x|+1)^{p /(p-1)},} & \text { if } \mu+\frac{n}{p}=n \\ \frac{1}{(1+|x|)^{(n-2 m) \wedge(\mu+n / p-2 m)},} & \text { if } \mu+\frac{n}{p} \neq n .\end{cases}
$$


Proof. Let $p>n / 2 m$ and $q \geq 1$ such that $1 / p+1 / q=1$. Let $a$ be a nonnegative function in $L^{p}\left(\mathbb{R}^{n}\right)$ and $\lambda<2 m-n / p<\mu$. Put $\varphi(x)=a(x) /(1+|x|)^{\mu-\lambda}|x|^{\lambda}$, then by the Hölder inequality, we have for each $x \in \mathbb{R}^{n}$

$$
\begin{aligned}
V \varphi(x) & \leq\|a\|_{p}\left(\int_{\mathbb{R}^{n}} \frac{d y}{|x-y|^{(n-2 m) q}(1+|y|)^{(\mu-\lambda) q}|y|^{\lambda q}}\right)^{1 / q} \\
& =\|a\|_{p}(I(x))^{1 / q} .
\end{aligned}
$$

Furthermore,

(i) if $|x| \leq 1$, we have by Lemma 2.12, that

$$
\begin{aligned}
I(x) & \leq \int_{B(x, 2)} \frac{d y}{|x-y|^{(n-2 m) q}|y|^{q \lambda^{+}}}+\int_{B^{c}(x, 2)} \frac{d y}{|x-y|^{(n-2 m) q}|y|^{\mu q}} \\
& \preceq \int_{B(x, 2)} \frac{d y}{|x-y|^{(n-2 m) q}|y|^{q \lambda^{+}}}+\int_{B^{c}(0,2)} \frac{d y}{|x-y|^{(n-2 m+\mu) q}} \\
& \preceq 1,
\end{aligned}
$$

(ii) if $|x| \geq 1$, we have

$$
\begin{aligned}
& I(x) \leq \int_{(|y| \leq 1 / 2)} \frac{d y}{|x-y|^{(n-2 m) q|y|^{\lambda q}}}+\int_{(|y| \geq 1 / 2) \cap(|x-y| \leq|x| / 2)} \frac{d y}{|x-y|^{(n-2 m) q|y|^{\mu q}}} \\
&+\int_{(|y| \geq 1 / 2) \cap(|x| / 2 \leq|x-y| \leq 2|x|)} \frac{d y}{|x-y|^{(n-2 m) q}|y|^{\mu q}} \\
&+\int_{(|y| \geq 1 / 2) \cap(|x-y| \geq 2|x|)} \frac{d y}{|x-y|^{(n-2 m) q|y|^{\mu q}}} \\
& \leq \frac{1}{|x|^{(n-2 m) q}} \int_{(|y| \leq 1 / 2)} \frac{d y}{|y|^{\lambda q}}+\frac{1}{|x|^{\mu q}} \int_{(|x-y| \leq|x| / 2)} \frac{d y}{|x-y|^{(n-2 m) q}} \\
&+\frac{1}{|x|^{(n-2 m) q}} \int_{(1 / 2 \leq|y| \leq 3|x|)} \frac{d y}{|y|^{\mid \mu q}}+\int_{(|x-y|>2|x|)} \frac{d y}{|x-y|^{(n-2 m+\mu) q}} \\
& \preceq \frac{1}{|x|^{(n-2 m) q}}\left\{\begin{array}{l}
\log (|x|+1), \quad \text { if } \mu+\frac{n}{p}=n \\
1, \quad \text { if } \mu+\frac{n}{p}<n
\end{array}\right. \\
& 1, \quad \text { if } \mu+\frac{n}{p}>n .
\end{aligned}
$$

By combining the above inequalities, we get the result.

Corollary 2.16. The class of functions $L^{\infty}\left(\mathbb{R}^{n}\right) /(1+|\cdot|)^{\mu-\lambda}|\cdot|^{\lambda}$ is included in $K_{m, n}^{\infty}\left(\mathbb{R}^{n}\right)$ if and only if $\lambda<2 m<\mu$. 
Proof. " $\Leftarrow$ " follows from Proposition 2.13.

“ $\Rightarrow$ " Suppose that the function $\varphi$ defined on $\mathbb{R}^{n}$ by $\varphi(x)=1 /(1+|x|)^{\mu-\lambda}|x|^{\lambda}$ is in $K_{m, n}^{\infty}\left(\mathbb{R}^{n}\right)$. Then by Proposition 2.10, we have $\int_{0}^{\infty} r^{2 m-1} \varphi(r) d r<\infty$. This implies that $\lambda<$ $2 m<\mu$.

Remark 2.17. Let $\lambda<2 m<\mu$ and $\varphi(x)=1 /(1+|x|)^{\mu-\lambda}|x|^{\lambda}$, for $x \in \mathbb{R}^{n}$, then by simple calculus, we obtain the following behaviour on the $m$-potential

$$
V \varphi(x) \sim \begin{cases}\frac{1}{(1+|x|)^{n-2 m}} \log (|x|+1), & \text { if } \mu=n \\ \frac{1}{(1+|x|)^{(n-2 m) \wedge(\mu-2 m)}}, & \text { if } \mu \neq n .\end{cases}
$$

\section{First existence result}

In this section, we aim at proving Theorem 1.4. The following lemmas are useful.

Lemma 3.1. Let $\varphi$ be a nonnegative function in $K_{m, n}^{\infty}\left(\mathbb{R}^{n}\right)$. Then we have

$$
\|V \varphi\|_{\infty} \leq \alpha_{\varphi} \leq 2^{n-2 m}\|V \varphi\|_{\infty}
$$

Proof. By (1.3) we obtain easily that $\alpha_{\varphi} \leq 2^{n-2 m}\|V \varphi\|_{\infty}$. On the other hand, by letting $|y| \rightarrow \infty$ in (1.24), we deduce from Fatou Lemma that $\|V \varphi\|_{\infty} \leq \alpha_{\varphi}$.

Lemma 3.2. Let $\varphi$ be a nonnegative function in $K_{m, n}^{\infty}\left(\mathbb{R}^{n}\right)$. Then for each $x \in \mathbb{R}^{n}$, we have

$$
V\left(\varphi G_{m, n}(\cdot, y)\right)(x) \leq \alpha_{\varphi} G_{m, n}(x, y)
$$

Proof. The result holds by (1.24).

In the sequel, let $q$ be a nonnegative function in $K_{m, n}^{\infty}\left(\mathbb{R}^{n}\right)$ such that $\alpha_{q} \leq 1 / 2$. For $f \in$ $\mathscr{B}^{+}\left(\mathbb{R}^{n}\right)$, we will define the potential kernel $V_{q} f:=V_{m, n, q} f$ as a solution for the perturbed polyharmonic equation (1.9).

We put for $x, y \in \mathbb{R}^{n}$,

$$
\mathscr{G}_{m, n}(x, y)= \begin{cases}\sum_{k \geq 0}(-1)^{k}(V(q \cdot))^{k}\left(G_{m, n}(\cdot, y)\right)(x), & \text { if } x \neq y \\ \infty, & \text { if } x=y\end{cases}
$$

Then we have the following comparison result.

Lemma 3.3. Let $q$ be a nonnegative function in $K_{m, n}^{\infty}\left(\mathbb{R}^{n}\right)$ such that $\alpha_{q} \leq 1 / 2$. Then for $x, y \in \mathbb{R}^{n}$, we have

$$
\left(1-\alpha_{q}\right) G_{m, n}(x, y) \leq \mathscr{G}_{m, n}(x, y) \leq G_{m, n}(x, y)
$$


Proof. Since $\alpha_{q} \leq 1 / 2$, we deduce from (3.2), that

$$
\begin{aligned}
\left|\mathscr{G}_{m, n}(x, y)\right| & \leq \sum_{k \geq 0}\left(\alpha_{q}\right)^{k} G_{m, n}(x, y) \\
& =\frac{1}{1-\alpha_{q}} G_{m, n}(x, y) .
\end{aligned}
$$

Furthermore, we have for $x \neq y$ in $\mathbb{R}^{n}$

$$
\mathscr{G}_{m, n}(x, y)=G_{m, n}(x, y)-V\left(q^{\mathscr{G}_{m, n}}(\cdot, y)\right)(x),
$$

which together with (3.2), imply that

$$
\begin{aligned}
\mathscr{G}_{m, n}(x, y) & \geq G_{m, n}(x, y)-\frac{\alpha_{q}}{1-\alpha_{q}} G_{m, n}(x, y) \\
& =\frac{1-2 \alpha_{q}}{1-\alpha_{q}} G_{m, n}(x, y) \\
& \geq 0 .
\end{aligned}
$$

Hence the result follows from (3.6) and (3.2).

Let us define the operator $V_{q}$ on $\mathscr{B}^{+}\left(\mathbb{R}^{n}\right)$ by

$$
V_{q} f(x)=\int_{B} \mathscr{G}_{m, n}(x, y) f(y) d y, \quad x \in \mathbb{R}^{n} .
$$

Then we obtain the following.

Lemma 3.4. Let $f \in \mathscr{B}^{+}\left(\mathbb{R}^{n}\right)$. Then $V_{q} f$ satisfies the following resolvent equation

$$
V f=V_{q} f+V_{q}(q V f)=V_{q} f+V\left(q V_{q} f\right) .
$$

Proof. From the expression of $\varphi_{m, n}$, we deduce that for $f \in \mathscr{B}^{+}\left(\mathbb{R}^{n}\right)$ such that $V f<\infty$,

$$
V_{q} f=\sum_{k \geq 0}(-1)^{k}(V(q \cdot))^{k} V f
$$

So we obtain that

$$
\begin{aligned}
V_{q}(q V f) & =\sum_{k \geq 0}(-1)^{k}(V(q \cdot))^{k}[V(q V f)] \\
& =-\sum_{k \geq 1}(-1)^{k}(V(q \cdot))^{k} V f \\
& =V f-V_{q} f .
\end{aligned}
$$

The second equality holds by integrating (3.6).

Proposition 3.5. Let $f \in L_{\text {loc }}^{1}\left(\mathbb{R}^{n}\right)$ such that $V f \in L_{\text {loc }}^{1}\left(\mathbb{R}^{n}\right)$. Then $V_{q} f$ is a solution (in the sense of distributions) of the perturbed polyharmonic equation (1.9). 
18 Solutions of polyharmonic equations in $\mathbb{R}^{n}$

Proof. Using the resolvent equation (3.9), we have

$$
V_{q} f=V f-V\left(q V_{q} f\right) .
$$

Applying the operator $(-\Delta)^{m}$ on both sides of the above equality, we obtain that

$$
(-\Delta)^{m}\left(V_{q} f\right)=f-q V_{q} f \quad \text { (in the sense of distributions). }
$$

This completes the proof.

Now, we are ready to prove Theorem 1.4.

Proof of Theorem 1.4. Let $c>0$. Then by $\left(\mathrm{H}_{2}\right)$, there exists a nonnegative function $q:=$ $q_{c} \in K_{m, n}^{\infty}\left(\mathbb{R}^{n}\right)$, such that $\alpha_{q} \leq 1 / 2$ and for each $x \in \mathbb{R}^{n}$, the map

$$
t \rightarrow t(q(x)-\varphi(x, t)) \text { is continuous and nondecreasing on }[0, c],
$$

which implies in particular that for each $x \in \mathbb{R}^{n}$ and $t \in[0, c]$,

$$
0 \leq \varphi(x, t) \leq q(x)
$$

Let

$$
\Lambda:=\left\{u \in \mathscr{B}^{+}\left(\mathbb{R}^{n}\right):\left(1-\alpha_{q}\right) c \leq u \leq c\right\}
$$

We define the operator $T$ on $\Lambda$ by

$$
T u(x):=c\left(1-V_{q}(q)(x)\right)+V_{q}[(q-\varphi(\cdot, u)) u](x) .
$$

First, we prove that $\Lambda$ is invariant under $T$. Indeed, for each $u \in \Lambda$, we have

$$
T u \leq c\left(1-V_{q}(q)(x)\right)+c V_{q}(q)(x) \leq c .
$$

Moreover, from (3.15), (3.4) and Lemma 3.1 we deduce that for each $u \in \Lambda$, we have

$$
T u \geq c\left(1-V_{q}(q)(x)\right) \geq c(1-V(q)(x)) \geq c\left(1-\alpha_{q}\right) .
$$

Next, we prove that the operator $T$ is nondecreasing on $\Lambda$. Indeed, let $u, v \in \Lambda$ such that $u \leq v$, then from (3.14) we obtain that

$$
T v-T u=V_{q}([(q-\varphi(\cdot, v)) v]-[(q-\varphi(\cdot, u)) u]) \geq 0 .
$$

Now, consider the sequence $\left(u_{k}\right)$ defined by $u_{0}=\left(1-\alpha_{q}\right) c$ and $u_{k+1}=T u_{k}$, for $k \in \mathbb{N}$. Then since $\Lambda$ is invariant under $T$, we obtain obviously that $u_{1}=T u_{0} \geq u_{0}$ and so from the monotonicity of $T$, we have

$$
u_{0} \leq u_{1} \leq \cdots \leq u_{k} \leq c
$$


So from (3.14) and the dominated convergence theorem we deduce that the sequence $\left(u_{k}\right)$ converges to a function $u \in \Lambda$ which satisfies

$$
u=c\left(1-V_{q}(q)(x)\right)+V_{q}[(q-\varphi(\cdot, u)) u](x)
$$

That is

$$
u-V_{q}(q u)=c\left(1-V_{q}(q)(x)\right)-V_{q}(u \varphi(\cdot, u))
$$

Applying the operator $(I+V(q \cdot))$ on both sides of the above equality and using (3.9) we deduce that $u$ satisfies

$$
u=c-V(u \varphi(\cdot, u))
$$

Finally, we claim that $u$ is a positive continuous solution for the Problem (1.6). To prove the claim, we use Lemma 2.4. Indeed, since $u \sim c$ on $\mathbb{R}^{n}$ and

$$
0 \leq u \varphi(\cdot, u) \leq c q
$$

we deduce that either $u$ and $u \varphi(\cdot, u)$ are in $L_{\text {loc }}^{1}\left(\mathbb{R}^{n}\right)$.

Now, from (3.24) we can easily see that $V(u \varphi(\cdot, u)) \in L_{\text {loc }}^{1}\left(\mathbb{R}^{n}\right)$. Hence $u$ satisfies (in the sense of distributions) the elliptic differential equation

$$
(-\Delta)^{m} u+u \varphi(\cdot, u)=f \quad \text { in } \mathbb{R}^{n}
$$

On the other hand, it follows from (3.25) that $u \varphi(\cdot, u) \in M_{q}$ and so by Proposition 2.8, we obtain that $V(u \varphi(\cdot, u))$ is in $C_{0}^{+}\left(\mathbb{R}^{n}\right)$.

This implies by (3.24) that $\lim _{|x| \rightarrow \infty} u(x)=c$, which completes the proof.

Remark 3.6. Let $c>0$ and $u$ be a solution of (1.8). Then we have by Theorem 1.4 that for each $x \in \mathbb{R}^{n}, 0 \leq u(x) \leq c$. Let $q$ be the nonnegative function in $K_{m, n}^{\infty}\left(\mathbb{R}^{n}\right)$ given in the proof of Theorem 1.4. Then we deduce from (3.24) and (3.25), that

$$
0 \leq c-u(x)=V(u \varphi(\cdot, u))(x) \leq c V(q)(x)
$$

Example 3.7. Let $p>n / 2 m$ and $a$ be a nonnegative function in $L^{p}\left(\mathbb{R}^{n}\right)$. Let $\lambda<2 m-n / p<$ $\mu$ and $\alpha, \beta$ be two nonnegative constants.

Put $q(x)=a(x) /(1+|x|)^{\mu-\lambda}|x|^{\lambda}$. Then, for each $c>0$, the following polyharmonic problem

$$
\begin{gathered}
(-\triangle)^{m} u+\beta u^{\alpha+1} q=0, \quad \text { in } \mathbb{R}^{n} \text { (in the sense of distributions) } \\
\lim _{|x| \rightarrow \infty} u(x)=c,
\end{gathered}
$$

has a positive continuous solution satisfying $c / 2 \leq u(x) \leq c$, provided that $\beta$ is sufficiently small. 
Moreover, by Remark 3.6 and Proposition 2.15, we have

$$
0 \leq c-u(x) \leq c\|a\|_{p} \begin{cases}\frac{1}{(1+|x|)^{n-2 m}} \log (|x|+1)^{p /(p-1)}, & \text { if } \mu+\frac{n}{p}=n \\ \frac{1}{(1+|x|)^{(n-2 m) \wedge(\mu+n / p-2 m)},} & \text { if } \mu+\frac{n}{p} \neq n .\end{cases}
$$

Remark 3.8. It is interesting to compare the asymptotics (3.29) with the results of Trubek [10], for the case $m=1$.

\section{Second existence result}

In this section, we aim at proving Theorem 1.5.

Proof of Theorem 1.5. Assuming $\left(\mathrm{H}_{3}\right)$ and $\left(\mathrm{H}_{4}\right)$, we will use the Schauder fixed point theorem. From (1.14), there exists $\eta>0$ such that

$$
h(t) \geq m_{0} t, \quad \text { for each } t \in[0, \eta] .
$$

On the other hand, let $\alpha \in\left(g^{\infty}, M_{0}\right)$, then by (1.15), there exists $\rho>0$ such that for $t \geq \rho$, we have $g(t) \leq \alpha t$. Put $\beta=\sup _{0 \leq t \leq \rho} g(t)$. So we deduce that

$$
0 \leq g(t) \leq \alpha t+\beta, \quad \text { for each } t \geq 0 \text {. }
$$

By Remark 2.14, we note that there exists a constant $\alpha_{1}>0$ such that

$$
\frac{\alpha_{1}}{(1+|x|)^{n-2 m}} \leq V q(x) .
$$

Let $a \in(0, \eta)$ and $b=\max \left\{a / \alpha_{1}, \beta /\left(1-\alpha\|V q\|_{\infty}\right)\right\}$. So we consider the closed convex set

$$
\Lambda=\left\{u \in C_{0}\left(\mathbb{R}^{n}\right), \frac{a}{(1+|x|)^{n-2 m}} \leq u(x) \leq b V q(x), \forall x \in \mathbb{R}^{n}\right\}
$$

Obviously by (4.3) we have that the set $\Lambda$ is nonempty. Next we define the operator $T$ on $\Lambda$ by

$$
T u(x)=\int_{\mathbb{R}^{n}} G_{m, n}(x, y) f(y, u(y)) d y .
$$

Let us prove that $T \Lambda \subset \Lambda$. Let $u \in \Lambda$, then by (4.2) we have

$$
\begin{aligned}
T u(x) & \leq \int_{\mathbb{R}^{n}} G_{m, n}(x, y) q(y) g(u(y)) d y \\
& \leq \int_{\mathbb{R}^{n}} G_{m, n}(x, y) q(y)[\alpha u(y)+\beta] d y \\
& \leq\left(\alpha b\|V q\|_{\infty}+\beta\right) V q(x) \\
& \leq b V q(x) .
\end{aligned}
$$


Moreover, since $h$ is nondecreasing, we deduce by (4.1) and (1.14) that

$$
\begin{aligned}
T u(x) & \geq \int_{\mathbb{R}^{n}} G_{m, n}(x, y) p(y) h(u(y)) d y \\
& \geq \int_{\mathbb{R}^{n}} G_{m, n}(x, y) p(y) h\left(\frac{a}{(1+|y|)^{n-2 m}}\right) d y \\
& \geq m_{0} a \int_{\mathbb{R}^{n}} G_{m, n}(x, y) \frac{p(y)}{(1+|y|)^{n-2 m}} d y \\
& \geq \frac{m_{0} a k_{m, n}}{(1+|x|)^{n-2 m}} \int_{\mathbb{R}^{n}} \frac{p(y)}{(1+|y|)^{2(n-2 m)}} d y \\
& =\frac{a}{(1+|x|)^{n-2 m}} .
\end{aligned}
$$

On the other hand, by (1.13), we have that for each $u \in \Lambda$

$$
f(\cdot, u) \leq g\left(b\|V q\|_{\infty}\right) q .
$$

This implies by Proposition 2.8 that $T u \in V\left(M_{q}\right) \subset C_{0}\left(\mathbb{R}^{n}\right)$. So $T \Lambda \subset \Lambda$.

Next, we prove the continuity of $T$ in $\Lambda$. Let $\left(u_{k}\right)$ be a sequence in $\Lambda$, which converges uniformly to a function $u \in \Lambda$. Then using (4.8) and $\left(\mathrm{H}_{3}\right)$, we deduce by Theorem 1.3 and the dominated convergence Theorem that for $x \in \mathbb{R}^{n}$,

$$
T u_{k}(x) \longrightarrow T u(x) \text { as } k \longrightarrow \infty .
$$

Now, since $T \Lambda \subset V\left(M_{q}\right)$, we deduce by Proposition 2.8 that $T \Lambda$ is relatively compact in $C_{0}\left(\mathbb{R}^{n}\right)$, which implies that

$$
\left\|T u_{k}-T u\right\|_{\infty} \longrightarrow 0 \quad \text { as } k \longrightarrow \infty
$$

Hence $T$ is a compact map from $\Lambda$ to itself. So the Schauder fixed point theorem leads to the existence of $u \in \Lambda$ such that

$$
u=V(f(\cdot, u))
$$

Finally by (4.8) and Lemma 2.4, we conclude that $y \rightarrow f(y, u(y))$ is in $L_{\text {loc }}^{1}\left(\mathbb{R}^{n}\right)$, which together with (4.11) imply that $u$ satisfies (in the sense of distributions) the elliptic differential equation

$$
(-\triangle)^{m} u=f(\cdot, u) \quad \text { in } \mathbb{R}^{n}
$$

This ends the proof.

Example 4.1. Let $p$ be a nonnegative function in $K_{m, n}^{\infty}\left(\mathbb{R}^{n}\right)$ and $0 \leq \alpha<1$. Then the following problem

$$
\begin{gathered}
(-\triangle)^{m} u+p(x) u^{\alpha}=0, \quad x \in \mathbb{R}^{n}, \\
\lim _{|x| \rightarrow \infty} u(x)=0,
\end{gathered}
$$


has a positive solution $u \in C_{0}\left(\mathbb{R}^{n}\right)$ satisfying for each $x \in \mathbb{R}^{n}$

$$
\frac{1}{(1+|x|)^{n-2 m}} \preceq u(x) \preceq V p(x) .
$$

\section{Third existence result}

In this section, we aim at proving Theorem 1.6.

Proof of Theorem 1.6. Let $c>0$ be the constant given by $\left(\mathrm{H}_{7}\right)$ and $c^{*}=c-\|V(q(\cdot, c))\|_{\infty}$. Let $\delta \in\left(0, c^{*}\right]$. We will use the Schauder fixed point theorem, so we consider the closed convex set

$$
\Lambda=\left\{u \in C\left(\mathbb{R}^{n} \cup\{\infty\}\right): \delta \leq u(x) \leq c, \forall x \in \mathbb{R}^{n}\right\}
$$

and we define the integral operator $T$ on $\Lambda$ by

$$
T u(x)=\delta+V(f(\cdot, u))(x) .
$$

First, we prove that $T \Lambda \subset \Lambda$. Let $u \in \Lambda$, then since $f$ is a nonnegative function, we have that $\operatorname{Tu}(x) \geq \delta$, for each $x \in \mathbb{R}^{n}$. Moreover by $\left(\mathrm{H}_{6}\right)$, we have for $x \in \mathbb{R}^{n}$,

$$
T u(x) \leq \delta+V(q(\cdot, u))(x) \leq c^{*}+V(q(\cdot, c))(x) \leq c .
$$

Furthermore by $\left(\mathrm{H}_{7}\right)$, since for all $u \in \Lambda, f(\cdot, u) \in M_{q(\cdot, c)}$, then it follows from Proposition 2.8 that $V(f(\cdot, u)) \in C_{0}\left(\mathbb{R}^{n}\right)$ and more precisely $T \Lambda$ is relatively compact in $C\left(\mathbb{R}^{n} \cup\{\infty\}\right)$. Therefore $T \Lambda \subset \Lambda$.

Next, let us prove the continuity of $T$ in $\Lambda$. Let $\left(u_{k}\right)$ be a sequence in $\Lambda$, which converges uniformly to a function $u \in \Lambda$. Since $f$ is continuous with respect to the second variable, we deduce by the dominated convergence theorem that for each $x \in \mathbb{R}^{n} \cup\{\infty\}$,

$$
T u_{k}(x) \longrightarrow T u(x) \text { as } k \longrightarrow \infty
$$

Now, since $T \Lambda$ is relatively compact in $C\left(\mathbb{R}^{n} \cup\{\infty\}\right)$, then

$$
\left\|T u_{k}-T u\right\|_{\infty} \longrightarrow 0 \quad \text { as } k \longrightarrow \infty
$$

Finally the Schauder fixed point theorem implies the existence of $u \in \Lambda$ such that

$$
u(x)=\delta+V(f(\cdot, u))(x), \quad \forall x \in \mathbb{R}^{n} .
$$

Using $\left(\mathrm{H}_{6}\right),\left(\mathrm{H}_{7}\right)$ and Lemma 2.4, we deduce that the function $y \rightarrow f(y, u(y))$ is in $L_{\text {loc }}^{1}\left(\mathbb{R}^{n}\right)$. So $u$ satisfies (in the sense of distributions) the elliptic differential equation

$$
(-\triangle)^{m} u=f(\cdot, u) \quad \text { in } \mathbb{R}^{n}
$$

Moreover since $V(f(\cdot, u)) \in C_{0}\left(\mathbb{R}^{n}\right)$, then by (5.6) it follows that $\lim _{|x| \rightarrow \infty} u(x)=\delta$. This ends the proof. 
Corollary 5.1. Assume that $q(x, t)=p(x) g(t)$, where $g$ is a nonnegative nondecreasing measurable function and $p$ is a nonnegative function in $K_{m, n}^{\infty}\left(\mathbb{R}^{n}\right)$. If the function $g$ satisfies either $g(t)=o(t)$ as $t \rightarrow 0$ or $g(t)=o(t)$ as $t \rightarrow \infty$, then the problem (1.19) has a positive solution $u \in C\left(\mathbb{R}^{n} \cup\{\infty\}\right)$.

Example 5.2. Among the equations of form (1.1), we have the Emden-Fowler equation of order $m$

$$
(-\triangle)^{m} u+p(x) u^{\alpha}=0, \quad \alpha>0, x \in \mathbb{R}^{n}, n>2 m,
$$

where $p \in K_{m, n}^{\infty}\left(\mathbb{R}^{n}\right)$.

(i) For the sublinear $(0<\alpha<1)$ or the superlinear $(\alpha>1)$ case, let $c>0$ such that

$$
\|V p\|_{\infty} c^{\alpha-1}<1
$$

Then applying Theorem 1.6, we deduce that for each $\delta \in\left(0, c\left(1-c^{\alpha-1}\|V p\|_{\infty}\right)\right)$, (5.8) with $\alpha \neq 1$ has a continuous positive solution $u$ in $\mathbb{R}^{n}$ with $\delta \leq u(x) \leq c$, for all $x \in \mathbb{R}^{n}$ and $\lim _{|x| \rightarrow \infty} u(x)=\delta$.

(ii) For the linear case $(\alpha=1)$. If $\|V p\|_{\infty}<1$, then applying Theorem 1.6, we deduce that for each $c>0$ and $\delta \in\left(0, c\left(1-\|V p\|_{\infty}\right)\right),(5.8)$ has a continuous positive solution $u$ in $\mathbb{R}^{n}$ with $\delta \leq u(x) \leq c$, for all $x \in \mathbb{R}^{n}$ and $\lim _{|x| \rightarrow \infty} u(x)=\delta$.

Remark 5.3. We improve in this section the Yin's result in [11]. Indeed, Yin proved in particular the existence of bounded positive solutions for the Emden-Fowler equation

$$
\triangle u+p(x) u^{\alpha}=0, \quad 0<\alpha \neq 1, x \in \mathbb{R}^{n}, n \geq 3
$$

provided that the function $p$ satisfies

$$
\int_{0}^{\infty} s \max _{|x|=s}\{p(x)\} d s<\infty
$$

However by taking $\lambda>(n-1) / 2$ and

$$
p(x)=p\left(x^{\prime}, x_{n}\right)=\frac{1}{\left(1+x_{n}^{2}\right)\left(1+\sum_{i=1}^{n-1} x_{i}^{2}\right)^{\lambda}}, \quad x \in \mathbb{R}^{n},
$$

then we have

$$
\max _{|x|=s} p(x) \geq p(0, s)=\frac{1}{1+s^{2}}
$$

which implies that (5.11) is not satisfied. On the other hand, we have that $p \in L^{\infty}\left(\mathbb{R}^{n}\right) \cap$ $L^{1}\left(\mathbb{R}^{n}\right) \subset K_{m, n}^{\infty}\left(\mathbb{R}^{n}\right)$. This implies by Corollary 5.1 that the Emden-Fowler equation (5.8) has a positive solution $u \in C\left(\mathbb{R}^{n} \cup\{\infty\}\right)$, for each $m \geq 1$. 


\section{Solutions of polyharmonic equations in $\mathbb{R}^{n}$}

\section{References}

[1] M. Aizenman and B. Simon, Brownian motion and Harnack inequality for Schrödinger operators, Communications on Pure and Applied Mathematics 35 (1982), no. 2, 209-273.

[2] I. Bachar, H. Mâagli, and N. Zeddini, Estimates on the Green function and existence of positive solutions of nonlinear singular elliptic equations, Communications in Contemporary Mathematics 5 (2003), no. 3, 401-434.

[3] H. Brezis and S. Kamin, Sublinear elliptic equations in $\mathbf{R}^{n}$, Manuscripta Mathematica 74 (1992), no. 1, 87-106.

[4] K. L. Chung and Z. X. Zhao, From Brownian Motion to Schrödinger's Equation, Grundlehren der Mathematischen Wissenschaften, vol. 312, Springer, Berlin, 1995.

[5] R. Dalmasso, Existence and uniqueness results for polyharmonic equations, Nonlinear Analysis, Ser. A: Theory Methods 36 (1999), no. 1, 131-137.

[6] H.-C. Grunau and G. Sweers, The maximum principle and positive principal eigenfunctions for polyharmonic equations, Reaction Diffusion Systems (Trieste, 1995) (G. Caristi and E. Mitidieri, eds.), Lecture Notes in Pure and Appl. Math., vol. 194, Dekker, New York, 1998, pp. 163-182.

[7] H. Mâagli and S. Masmoudi, Existence and asymptotic behaviour of large solutions of semilinear elliptic equations, Potential Analysis 17 (2002), no. 4, 337-350.

[8] __ Positive solutions of some nonlinear elliptic problems in unbounded domain, Annales Academiæ Scientiarium Fennicæ. Mathematica 29 (2004), no. 1, 151-166.

[9] H. Mâagli and M. Zribi, Existence and estimates of solutions for singular nonlinear elliptic problems, Journal of Mathematical Analysis and Applications 263 (2001), no. 2, 522-542.

[10] J. Trubek, Asymptotic behavior of solutions to $\Delta u+K u^{\sigma}=0$ on $\mathbf{R}^{n}$ for $n \geqslant 3$, Proceedings of the American Mathematical Society 106 (1989), no. 4, 953-959.

[11] Z. Yin, Bounded positive solutions of Schrödinger equations, Applied Mathematics Letters 17 (2004), no. 5, 527-533.

[12] Q. S. Zhang and Z. X. Zhao, Singular solutions of semilinear elliptic and parabolic equations, Mathematische Annalen 310 (1998), no. 4, 777-794.

[13] Z. X. Zhao, Green function for Schrödinger operator and conditioned Feynman-Kac gauge, Journal of Mathematical Analysis and Applications 116 (1986), no. 2, 309-334.

[14] On the existence of positive solutions of nonlinear elliptic equations-a probabilistic potential theory approach, Duke Mathematical Journal 69 (1993), no. 2, 247-258.

Habib Mâagli: Département de Mathématiques, Faculté des Sciences de Tunis,

Campus Universitaire, 2092 Tunis, Tunisia

E-mail address: habib.maagli@fst.rnu.tn

Malek Zribi: Département de Mathématiques, Faculté des Sciences de Tunis, Campus Universitaire, 2092 Tunis, Tunisia

E-mail address: malek.zribi@insat.rnu.tn 


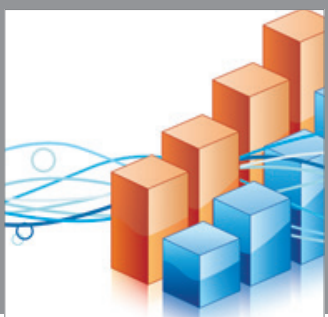

Advances in

Operations Research

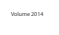

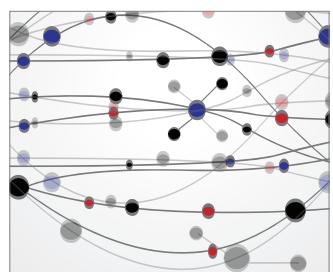

\section{The Scientific} World Journal
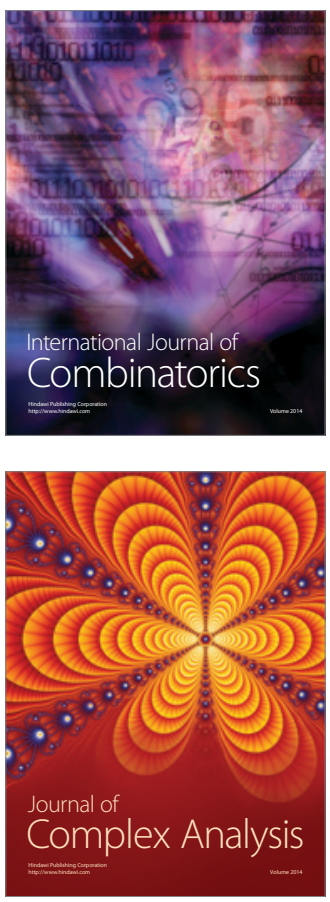

International Journal of

Mathematics and

Mathematical

Sciences
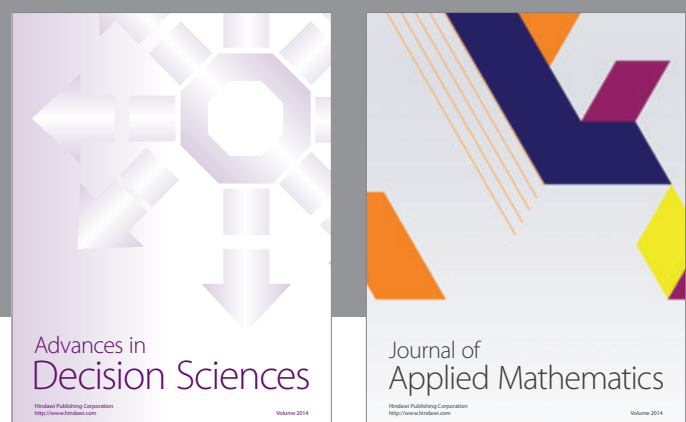

Journal of

Applied Mathematics
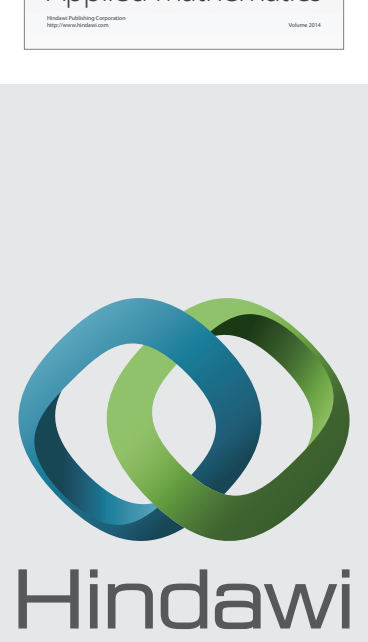

Submit your manuscripts at http://www.hindawi.com
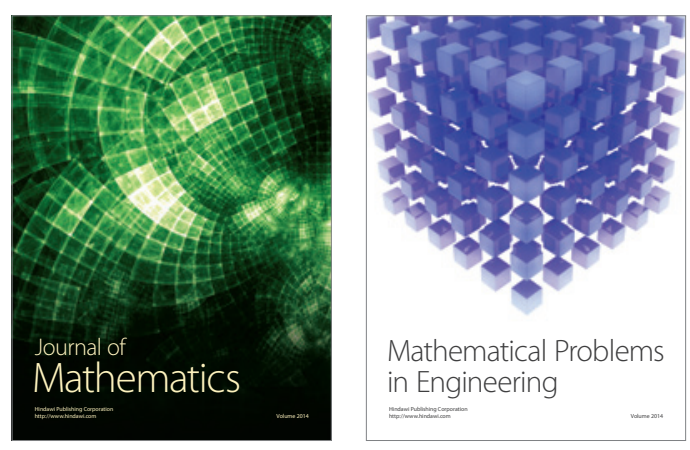

Mathematical Problems in Engineering
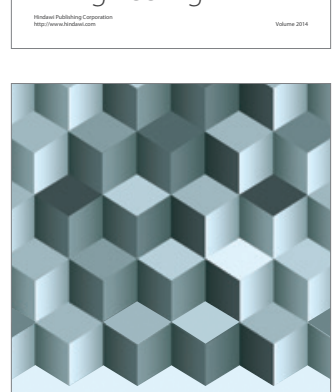

Journal of

Function Spaces
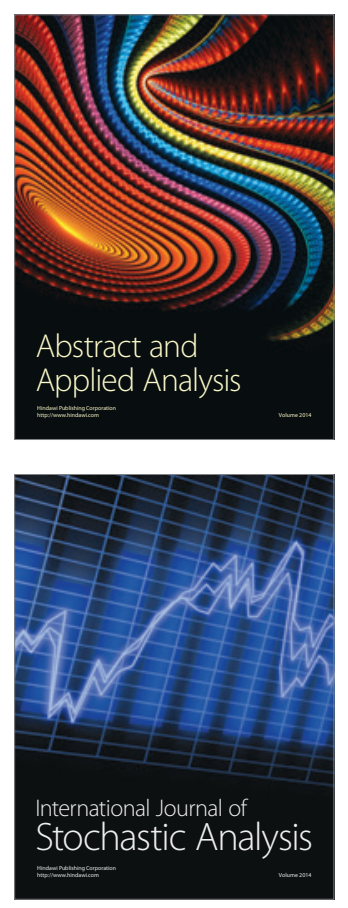

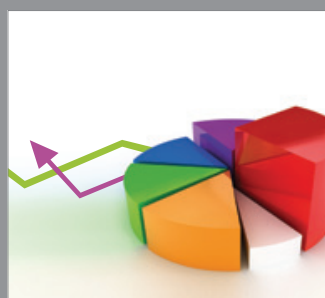

ournal of

Probability and Statistics

Promensencen
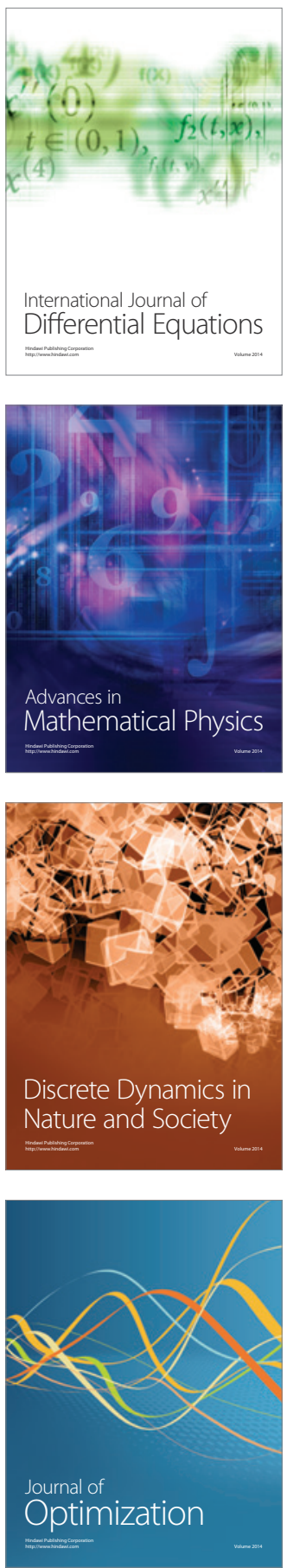